\title{
Demonstrationsabende im Alten Allgemeinen Krankenhause St. Georg, Hamburg.
}

\author{
Demonstrationsabend vom 20. Oktober 1906.
}

I. Arning, als nunmehriger Oberarzt der Abteilung für Haut - und Geschlechtskrankheiten dieses Instituts, stellt vor: eine 35jährige unverheiratete Frau mit Erythrodermia exfoliativa universalis chronica, den die Wiener Schule vielleicht für einen nicht ganz ausgeprägten Fall von Pityriasis rubra Hebrae halten würde; doch fehle hier manches, und es seien diese Fälle als ganz individuell, jeder für sich bestehend, aus dem großen Topf der Erythrodermien herauszaheben, und von ihnen existiere bis jetzt erst eine verhältnismäßig kleine Serie. Patientin, die einen durchaus gesunden, sonst kräftigen Eindruck macht (fieberfrei), hat seinerzeit eine Bubooperation linkerseits durchgemacht und sei anch sonst bis auf diese vor 16 Jahren aufgetretene Hauterkrankung gesund gewesen. Die erste Erscheinung bildete eine scheinbar barmlose "Flechte" in der linken Ellenbogenbeuge, dieselbe breitete sich langsam über den ganzen Körper und den Extremitäten (exklusive Hände, die auch jetzt frei sind) aus; die sonst beschriebene Affektion der Nägel fehlt und das Gesicht ist ebenfalls frei von der Schilferung und von Rhagaden, die besonders reich an Brüsten, Bauch und Rücken ins Auge fallen. Bei sonst ungestörtem Allgemeinbefinden - so daß Patientin bis vor kurzem nngestört ibrer Arbeit nachgegangen ist (Masseuse) - bestebt intensives Frostgefühl (besonders am Rücken) und Unvermögen spontan zu schwitzen. Arning indentifiziert diesen Fall mit den von Brocq und Vidalals Dermatitis exfoliatives généraliséeschroniques bezeichneten und faßt ihn als toxische Hauterkrankung auf. Die Franzosen unterscheiden bekanntlich 8 hierher gehörige Formen: a) akute, benigne; 
b) subakute und c) chronische. In einem zur Sektion gekommenen Fall ad a) haben sich gar keine organischen, auf die Hautkrankheit zu beziehenden Veränderungen gefunden, und ebenso fehlen in den za $b$ ) und c) zu rechnenden, im allgemeinen in $5-6$ Jahren verlaufenden Affektionen, Störungen des Allgemeinbefindens. Auch der Blutbefund ergibt in diesem Falle nichts von der Norm Abweichendes.

Zur Differentialdiagnose stehen Psoriasis (mit typischer Blutung nach Abkratzen der Schuppen) und Pemphigus (mit feuchtem Untergrund nach leichter, abziehender Entfernung der Haut oder der Blasen). Starke Pigmentierungen der abgeheilten Partien. Zwischen den mit trocknen, dünnen Lamellen bedeckten kranken Partien findet sich vollkommen gesunde Haut; es treten neue Schübe oft auch auf alten Herden auf. Haarboden gsund, Pubes- und Achselhöhlenhaare ebenfalls. Mikroskopischer Befund steht noch aus. Therapie einstweilen grobe Dosen Arsen.

II. Wilbrand einen Fall von Spätlues (8 Jahre) aus der Arningschen Abteilung bei einem Mann, die sich ophtbalmoskopisch als $\mathrm{S}$ e h n ervena trop b ie (links) präsentierte ; die ganz kolossale Einschränkung des Gesichtsfeldes und die dann in kürzester Zeit unter spezifischer Behandlung eingetretene, beinahe totale Rekonstruktion desselben wird in projizierten Bildern demonstriert. Früher rubrizierten derartige Fälle unter die allgemeine Diagnose „progressive genuine Sehnervenatrophie" und kamen nicht zur Heilung; jetzt seien diagnostisch davon ausgeschieden Perineuritis optica luetica, ferner Sehnervenatrophie bei multipler Sklerose und hysterische Asthenopie und S. N.-Atrophie bei Psendotabes als Perineuritis. Bei durchaus günstig zu stellender Prognose müsse man in allen diesen Fällen energisch und schnell therapeutisch vorgehen. W. demonstriert Projektionsbilder derartig affizierter Nerven.

Primäre Degeneration käme nur bei Tabes und Paralyse vor, alles übrige sei als Neuritis mit Druckatrophie aufzufassen. So auch der hier demonstrierte Fall, bei dem es sich um einen retrobulbären Herd handelt.

III. Albers-Sehönberg: a) mit einem neuen, billigen Apparat (Wiesbaden) hergestellte stereoskopische Röntgenaufnahmen; der Vorzug des betreffenden Apparats sei erstens dessen Billigkeit (zirka 35 Mark) und zweitens die durch ihn gegebene Möglichkeit, sofortiger Herstellung der Stereoskopie nach R.-Aufnahmen;

b) ein in 11 Röntgen-Sitzungen (à 6 Min.) von Haselnußgröße bis auf den Umfang einer kleinen Erbse reduziertes Cancroid an der linken Schläfe eines 66jähr. Mannes. A.-Sch. taxiert nach dem bisherigen günstigen Verlauf, daß nur noch etwa 4 Sitzungen à 6 Min. bis zur definitiven Beseitigung des in glattnarbiger, reaktionsloser Haut sitzenden Restes nötig sein werden. Im Nebenraum waren mehrere Mikroskope mit Präparaten von Spirochaete pallida aufgestellt. 
Demonstrationsabend vom 17. November 1906.

Wiesinger schildert eingehend den Normalverlauf einer Perityphlitisoperation und hebt rühmend die Methode der Hautnaht (nicht durch Catgut oder Seide), sondern durch Aluminiumbronze hervor; A. Br. könne sich nicht inbibieren und ließe deshalb nicht so erhebliche Stichnarben zurück (Demonstration).

Pasehen demonstriert folgenden Fall (Autoreferat): 47jähriger Mann. 1884 Primäraffekt. Sekundäre Affektionen nicht beobachtet. Seit 1887 sehr viel Kopfschmerzen; wegen Blutarmut von verschiedenen Ärzten Eisen. 1889 und 1892 wegen Hydrops genau punktiert. 1893, 5./I. Schwindel, leichte Unbesinnlichkeit, am nächsten Tage rechts Parese. Pulsan der rechten Radialis und Brachialis fehlt. Über dem Manubrium sterni weiches systolisches Blasen. Herztöne rein. Unter energischer Schmierkur und Jodkali gehen die Lähmungserscheinungen zurück. Juni 1893 Punktion des r. Kniegelenks wegen Hydrops genu mit nachfolgender Sublimatauswaschung. Seit der Zeit keine Gelenkschwellungen wieder, keine Kopfschmerzen. In den nächsten Jahren Jodkali; dazwischen auch Schmierkur. Bis 1898 gut. Sehr schwere Erkrankung an Aortitis mit heftigsten Schmerzen und Beklemmungen. Morphium in großen Dosen subkutan, dann wieder Schmierkur resp. Jodkalium. Die Oppressionserscheinungen verschwinden allmählich; jetzt hat Patient nur gelegentlich das Gefühl von Druck auf der Brust. Seit zirka 3 Jahren Schmerzen im r. Fuß, Schwellung des Gelenks; Injektion von Jodoformglyzerin ohne Erfolg (Sick). Frühjahr 1906 Zittmann, später Schmierkur, ohne Erfolg; Pat. kann nur mit den größten Schmerzen kurze Strecken gehen. Carotispuls links fehlt! Subklavia links über der Clavicula fühlbar mit deutlichem Schwirren. Radialpuls r. fehlt !

Status praesens: 17./XI. 1906: Puls an der r. Radialis und Brachialis sowie 1. Carotis fehlt. Deutliches Pulsieren der 1. Subclavia über der 1. Clavicula. Herzton 2 Finger nach außen von der Mamilla und nach unten. Durchleuchtung ergibt Querstellung des Herzens; Spitze sehr beweglich.

Die Ursache für diese Symptome ist eine Sklerose der Aorta; Verziehung der Abgangsstellen der r. Subclavia (vielleicht für sich entspringend aus dem arcus aortae, oder Kompression durch Aneurysma der r. Carotis und der l. Carotis, Aneurysma der Subelavia. Als Ursache für die Schmerzen und Funktionsstörungen im $r$. Fulgelenk fand sich auf der Röntgenplatte eine Knorpelspange auf dem Calcaneus, die die Bewegungen im Fußgelenke hindert.

(Hahn spricht sich gegen den syphilitischen Charakter der FuBgelenkserkrunkung aus, es scheine ihm hier (das Röntgenbild wird 
demonstriert) eine primäre Erkrankung der Knorpelscheibe vorzuliegen und nicht ein ausgeheiltes Fußknochengummi.)

Maess (an Engel-Reimers Stelle mit der Oberaufsicht $\ddot{u} b e r$ die hiesige Puellen.Behandlung betraut) demonstriert:

1. an einem 16jährigen Mädchen (Primäraffekt März 1906) ein durch seine Deutlichkeit und Lokalisation auffallendes Leucoderma syphilitic.; bei Lenc. colli hat sich dieses Hautphänomen auf dem Abdomen, den Flanken und dem Rücken (speziell im Kreuz) etabliert. (Ausgebreitete Alopecia diffusa capillitii.)

2. bei einem Mädchen (Primäraffekt 1900), welches wiederholt spezif. Kuren durchgemacht hat, ein differentialdiagnostisch interessantes guirlandenartiges Syphilià anden Vorderarmen und an den Beinen.

Sudeck: 1900 und 1902 habe Klemperer (in der "Therapie der Gegenwart") auf das Bild akuter Lebersyphilis aufmerksam gemacht: bei hohem Fieber plötzlich einsetzende Schmerzen im Epigastr. r. etc. Einen analogen Fall beobachtete Sudeck; dieser Fall kam unter hohem Fieber ad exitum, leider nicht zur Autopsie; plötzlich im Epigastr. auftretende Beschwerden, Auftreibung der Därme und Ascites; Leber nicht abzugrenzen, sehr hyperämisch und wesentlich vergrößert und zeigt beim Betasten auf vielen Stellen kleine Tumoren von zirka Haselnußgröße. Man konnte an subphreaischen Abszeß (auf dem r. Rippenbogen) oder an Leukämie oder an Gummiknoten denken.

Hecite demonstriert er einen solchen Fall im Genesungsstadium: 39jährige Frau, Nachts akut erkrankt an Schmerzen unter der Brust bis tief in den Leib hinein ausstrahlend; dieselben hatten gut 2 Tage lang angehalten, als Pat. aufgenommen wurde; auch hier differ. diagn. eventuell subphrenischer Abszeß; Schwellungen in der Lebergegend, besonders hart von außen anfühlbar und Druckschmerzen; die Ränder der Schwellung durch aufgetriebenen Darm überlagert; die Größe des vermeintlichen Tumors wiedersprach der Auffassung einer Gallenblasengeschwulst, ebenso einem Echinococcusabszeß. S. dachte in Anlehnung an oben Gesagtes an akute Hepatitis. Es fand sich außerdem daselbst ein pleuritisches Exs udat serösen Inhalts, ohne Bakterien, von zirka $700 \mathrm{~cm}^{3}$ Inhalt. S. gab reichliche subkutane Sublimatinjektionen; gleich darauf Abschwellen der Leber und Zurückgehen der Temp. zur Norm.; dennoch glaubt $\mathbf{S}$. aber den Erfolg nicht auf die spezif. Therapie, sondern eher auf ein Herabrücken der Leber durch das Abfallen des pleuritischen Exsudats [wie auch (z. B. bei Beurteilung der Klempererschen Fälle) Rosenbach] zurückführen zu müssen und wenn auch das nicht, so noch eher an eine akute Hepatitis, die schon spontane Neigung zum Abklingen zeigte, wobei der $\mathrm{Hg}-\mathrm{Kur}$ wohl ein erheblicher Anteil an der Heilung zugesprochen werden mag. - Die Anamnese ergab quoad Lues keine Anhaltspunkte.

Edleffsen tritt für die Iuetische Natur der Affektion ein. 


\section{Demonstrationsabend vom 15. Dezember 1906.}

Es demonstrieren:

1. Ringel ein zirka 6jähriges Mädehen, bei der er direkt unter der Achselhöhle (r.) ein einkammeriges Lymphangiom - er glaubt kongenitalen Ursprungs - operativ (durch Exstirpation) entfernt hat; bis jetzt sind - s. W. n. - solch ange eb or e ne Cystome nur am Halse beschrieben worden, doch hat W i esing er (am selben Krankenhause) neulich einen ganz analogen Fall auf seiner Abteilung operiert. Außer kongenital kämen ja derartige Bildungen auch auf traumatischem Wege zu stande, doch seien dieselben dann anders lokalisiert, so z. B. am Oberschenkel ( $N$ ordmann), oder durch Stoß ans Knie daselbst zu finden.

Außerdem finden wir an diesem Mädchen am Übergang der Kopfhaut auf den Helixrand (1.) oben einen kleinen, in der Haut liegenden und mit derselben verschieblichen Tumor, in seiner Wölbung von knapp Erbsengröße und unregelmäßiger Oberfläche, welchen R. ebenfalls für ein Lymphadenom hält, welches wohl in kongenitalem Zusammenhang mit der oben erwähnten Affektion stehe. - Wiesinger bestätigt die Diagnose des von ihm operierten absol. analogen Falles und macht auf die große Neigung zu Blutungen bei diesen Tumoren aufmerksam. Die durch Trauma entstandenen gleichartigen Bildungen reihen sich aber s. A. n. nicht an die auf kongenitalem Wege zu stande gekommenen an, sondern seien - wie die Franzosen sagen - dureh Décollement (Verschiebung) (eigentlich: Losgehen des Leimes. Ref.) der Haut zu erklären; diese verschwänden denn auch sehr rasch auf z. B. Jodinjektionen oder dergl. Therapie hin und brauchten nicht operiert zu werden.

2. Sinell ein 8jähriges Mädchen mit zerfallenem Tonsillargummi (1.) zur Diagnose; von der Mutter wisse er nur, daß sie vor einiger Zeit eine Schwellung an der Vulva gehabt habe. In der Dis= kussion macht Arning noch besonders auf die Formation der Schneidezähne des Mädchens aufmerksam und bestätigt die Diagnose.

3. Ma es einen Fall von Lupus syphiliticus (ulzeröses III. Syphilid) der Nasenspitze bei einer bereits vielfach mit Quecksilber und Jod vorbehandelten Puella, welcher seit 3 Wochen bestehen soll und bereits so viel zerstört habe, daß man nach eventueller Heilung durch energisches traitment mixte an Plastik werde denken müssen; mißlich sei aber die Prognose trotz der Plastik gerade bei diesen tertiären Zerstörungen.

4. Voretzsch (für Albers, Schönberg), ein in 22 RöntgenSitzungen à 3 Minuten im Laufe von 3 Monaten geheiltes Cancroid der Haut in der Mitte des Jochbeins 1.; 61jähriger Mann; von der nußgroß gewesenen Affektion sei nur noch ein schwacher Pigmentfleck übrig. Bei der Nachprüfung durch die Corona entstehen starke $Z$ weifel über 
die definitive Heilung, da man deutlich ein oder mehrere Knötchen in der Haut daselbst füblen kann (Deneke und Wiesinger), und Wiesinger macht gerade deswegen darauf aufmerksam, daß ein chirurgisches, absolut vollständiges Heilungsresultat in so einem Fall in einer kurzen Sitzung erzielt werden könne und für den Patienten zweifellos radikaler (und billiger! Ref.) sei.

5. Harmsen a) (für Arning) Favus am Skrotum eines jungen Maurers (wurde vor kurzem durch Arning im ärztl. Verein hierselbst vorgestellt); seit zirka $3^{1 / 2}$ Wochen befindet sich Patient wegen Akne des Gesichts im Krankenhaus und diese Affektion bestünde zirka 2 Wochen; die Ätiologie sei nicht ganz klar, da Pat. weder ein Kataplasma aufs Skrotum erhalten habe, noch eine familiäre Infektionsquelle nachweisbar sei; aber es hätten sich angeblich Mäuse in seinem Hause befundea. Pla u t berichtete bei dieser Gelegenheit im ärztl. Verein über die sicherste und einfachste Art davon zu machender Kulturen. Das ist nun gesebehen und ein projiziertes, sehr gelungenes Diapositiv derselben zeigt uns nun nach 2 Tagen schon entstandene Sprossungen (auch mikroskop. Präparat davon aufgestellt). 1897 habe Leik 7 bisher beobachtete Fälle von Favus des Skrotums zusammengestellt und soviel H. wisse, sei dieses also nun ein achter. (1898. 19. Apri) in der Wien. derm. Gesellschaft Dentsch (Abteilung Lang) Favus am Skrotum demonstr., referiert im Arch. f. Derm. und Syph. 1898. XLV. 412. - Ref.)

In der Diskussion betont Arning, daß dieses wohl sicher ein von Mänsen auf den Menschen übertragener Favus sei, da 1. nur ein solcher so schnell wachse, wie es hier in der Kultur der Fall gewesen sei, und 2. spräche auch der klinische Befund, daß sich nirgends ein Entzündungshof um die (schönen gelben) Skutula herum fände, dafür. Die Therapie sei hier - ganz im Gegensatz zum Favus der behandelten Kopfhaut, wo der Pilz tief in die Wurzelscheide der Haare eindringe - sehr erfolgreich, zumal das Skrotum dieses jungen Menschen so gut wie gar keine Haare aufweise, so dab er in zirka 14 Tagen unter Naphthol oder Chrysarobin eine vollständige Heilung zu erzielen hoffe.

b) Einen besonders schönen Fall von Vitilig o bei einem 14jähr., wegen allgemeiner Pediculosis aufgenommenen Jungen; Pat. habe die Fleckung der Haut angebl. erst vor 2 Jahren bemerkt, doch sei wegen der enormen Ausbreitung und wegen der sehr starken Pigmentanhäufungan den Rändern anzunehmen, daß sie schon wesentlich länger bestände; Ätiologie dunkel. Besonders präzise ist hier die Symmetrie der Affektion an Ober- und Unterschenkeln, am Penis, den Armen und speziell den Ellenbogen innegehalten bei sehr deutlich gezeichneter Konvexität der Ränder nach der gesunden Seite der Haut hin. Im übrigen ist Pat. ein durchaus wohlgebauter und gesunder Mensch. 


\section{Demonstrationsabend vom 20. April 1907.}

Meyer-Delius (Arningsche Abteilung) demonstriert:

1. Einen Gonorrhoiker, der während seines $2 \frac{1}{2}$ monatlichen Krankenhausaufenthaltes eine sehr ungewöhnliche Komplikation darbot. In verschieden großen Zwischenräumen, meist alle 4 Tage, doch auch häufiger, setzten hohe, bis $40.9^{\circ}$ a nsteigende Fieber at tacken ein, mit denen gleichzeitig, über Rumpf und Extremitäten verstreut, ohne besondere Prädilaktionsstellen ein makulöses oder papulöses Exanthem auftrat, das mit Aufhören des Fiebers, gewöhnlich schon am folgenden Tage, wieder verschwand. Fast jeder Anfall war ferner mit der Erkrankung irgend eines Gelenkes, eines Knies, eines Hand-, Kiefer-, Phalangealoder Rippengelenks verbunden, zuletzt trat vikariierend hierfür auch eine Myositis in den Muskelbäuchen der Extensorengruppe am rechten Vorderarme auf. Diese Affektionen verloren ihre Schmerzhaftigkeit ebenfalls mit dem Fieberabfall und waren wenige Tage darnach völlig verschwunden. Die Thatersuchung des Blutes während des Fieberanstieges und des Erythemknoten war bakteriologisch, mikroskopisch und kulturell, negativ. Vermutlich müssen alle diese Erscheinungen auf Wirkang des Toxins der Gonokokken zurückgeführt werden. Sehr auffallend war, daß der Patient sich während der schweren Fieberanfälle (immer über $39^{\circ}$ ) subjektiv fast gar nicht beeinträchtigt fühlte. Die Therapie war völlig machtlos, nach 10tägiger fieberfreier Periode bei geheilter Gonorrhoe wurde der Patient auf seinen Wunsch, da er sich, wie gesagt, durchaus nicht der Krankenhauspflege bedürftig fühlte, entlassen.

2. Ebenfalls auf Wirkung des Toxins der Gonokokken muß waurscheinlich die Balanitis circinata (zu unterscheiden von der durch Spirochaete refringens bedingten Balanitis erosiva circinosa) zurückgeführt werden, die Jadassohn in den "Komplikationen der Gonorrhoe" in der deutschen Klinik bei den hyperkeratotischen Exanthemen erwähnt, und die scheinbar nur in Begleitung von gonorrhoischer Arthritis und Conjunetivitis toxica vorkommt. Ein typischer, hochgradiger Fall bei einem gonorrhoischen Polyarthritiker wird demonstriert. Die Glans ist fast völlig von kaum linsengroßen, runden, bröckeligen Hornsehildern bedeckt, unter denen eine rings von einem leicht erhabenen Wall umgebene Frosion verborgen ist. Sind derartig hochgradige Balanitiscircinata-Formen anscheinend etwas seltenes, so sind mildere Formen bei Arthritikern gonorrhoischer Ätiologie entschieden etwas häufiges, was dadurch bewiesen wird, daß gleichzeitig 3 andere Patienten gezeigt werden können, bei denen die gleiche Affektion in den verschiedensten Intensitätsgraden vertreten ist. Am häufigsten zeigt sich diese Balanitis in Gestalt von senfkorn- bis linsengroßen Kreisen, deren Rand leicht aufgeworfen, aus grauweißlichen Körnchen zusammengesetzt ist, während das Zentrum eingesunken, manchmal von dünner Epidermisschuppe 
bedeckt ist, unter der eine oberflächliche Erosion zu Tage tritt. In diesen Effloreszenzen sind weder Gonokokken noch Spirochaete refringens nachzuweisen. Alle 4 demonstrierten Patienten sind Arthritiker, einer leidet gleichzeitig an einer Conjunctivitis toxica. Bei dreien von ihnen. inkl. dem hochgradigen Falle, fehlen andere hyperkeratotische Erscheinungen, bei dem vierten aber sind zugleich Verunstaltungen der Zehennägel und eine eigentümliche Veränderung der Fußsohlen vorhanden. Beide Fubsohlen, mit sehr derbem Hornlager versehen, sind von zahllosen reißzweckengroßen, strohgelben, zunächst von einem roten Hof umgebenen Epidermisabhebungen übersät, deren Inhalt eine krumelige, atheromatöse Detritusmasse ohne Bakteriengehalt ist. Nach Eröffnung der Blasen und Entleerung ihres Inhalts kommt der leicht entzündete Papillarkörper zum Vorschein. Diese Affektion, wie auch die Balanitis circinata gonococco-toxica heilten ab, spontan mit der Gonorrhoe, waren vorher aber therapeutisch wenig zu beeinfinssen.

3. Demonstration eines nicht gonorrhoischen chronischen Arth ri. tikers, bei dem eine Psoriasis vulgaris einen außerordentlich stark schuppenden Charakter zeigt, so daß also auch hier ein gleichzeitiges Vorkommen von Gelenkaffektion und Hyperkeratose zu verzeichnen ist.

Demonstrationsabend vom 15. Juni 1907.

Saenger demonstriert:

a) Ein 19 jähriges, sonst nervengesundes $M a ̈ d c h e n$ mit Herpes zoster, welcher seltener Weise alle $3 \ddot{A}$ ste des Nerv. trigeminus zugleich befallen hat (Analogie zu einem von Moers (?) beobachteten Fall). Die Affektion begann am 8./VI. mit Übelkeitsgefühl; es traten dann zuerst am Kinn die jetzt noch bestehenden Bläschen auf, dann wurde die Nase und zuletzt - am reichlichsten - das Gebiet des frontalis (speziell supraorbitalis) und des naso-ciliaris befallen; im ganzen streng rechtsseitig lokalisiert, nur am Kinn noch einzelne Übergriffe auch auf die linke Seite. Die Cornea ist freigeblieben, denn es besteht keine Areflexie, wenn auch leichte Herabminderung des Cornealreflexes.

b) 15jährigres imbecilles Mädchen mit Assymmetrie des Gesichtes zu Gunsten der r. Hälfte, Hemiatrophie faciei, Ptosis links und hy st e r is ch er A ma urose links; er bedeckt das r. Auge und veranlaBt Patientin einen Schlüsselbund zu holen, den er ca. 5 Meter von ihr entfernt auf einem Tiseh deponiert hat; das geschieht prompt. Mit dem Stereoskop warde bei ihr auch eine Amblyopie festgestellt, denn das angeblich nicht sebende Auge sollte auch im Stereoskop nicht sehen, aber trotzdem sah es! 
Delbanco (Gastdemonstration):

a) $1^{1} / 2$ jähriger Knabe mit extragenitalem Primäraffekt an der Oberlippe und im Filtrom nasi; deutlich - besonders an den Oberschenkeln - ausgesprochene Roseola und im Roseolenblut (Serum) (Paschen) Spirochaete pallida nachzuweisen, im Primäraffekt nicht (derselbe blutete stark nach Entfernung des Schorfs). Seine (ihn begleitende) Mutter hat ihn gestillt ohne infiziert zu werden; es läßt sich näher nichts Spezifisches nachweisen und der Vater sei ebenfalis absolut gesund.

b) Junger Mann mit I ch thyo sis nitida (d'A libert) und Primäraffekt; die Ichthyosis habe die Roseola wohl nicht aufkommen lassen, sie konnte wohl durch die so stark verdickte Oberfläche nicht durchschimmern. Delbanco sei gespannt, ob sich auch hier wird beobachten lassen, daß ein stark auftretendes akutes (luetisches) Exanthem die Ichthyosis eventuell zur Heilung bringen würde, wie es ihm von anderen akuten Exanthemen bekannt sei.

In der Diskussion erzählt Jolasse von einer Ichthyosis, welche durch ihn reichlich mit $R$ esor cin behandelt (und angeblich geheilt) wurde und bei der sich während des Gebrauchs besagten Medikamentes eine Psychose ausbildete; er fragt an, ob von den Anwesenden Ähnliches beobachtet worden sei? Delbanco weist darauf hin, daß man erfahrungsgemä $B$ bei stark reduzierenden Mitteln in der dermatologischen Praxis (Pyrogallus ete) überhaupt gut tue, zugleich Salzsäure zu geben, and daß es wohl möglich sei, daß dadurch bereits mancher Intoxikation vorgebengt worden sei; er glaube für den Fall Jolasse sicher an den Zusammenhang zwischen der dermatologischen Therapie und der Psychose (Orthoform).

Meyer-Delius (aus der Arningschen Abteilung) demonstriert einen jungen, sonst organisch vollkommen gesunden Kellner, der vor zirka 4 Wochen am Bein an einer (angeblich harmlosen) Flechte (in Güstrow) erkrankt sei; der behandelnde Arzt habe ibm dagegen eine "weiße" (?) Salbe gegeben und infolgedessen sei nunmehr schnellstens die Entwickelung einer über den ganzen Körper - inklusive Gesicht und Kopf - verbreiteten Dermatitis vor sich gegangen; heute ist der ganze Patient wie in ein weißes $S$ chuppenkleid gehüllt, welches aus kleinkalibrigen, schneeweißen und dicken einzelnen Lamellen besteht, die lose auf der dunkelrosa gefärbten und etwas verdickten Oberhaut aufsitzen, so daß Morgens das Bett und die Umgebung desselben wie besät mit Schuppen sei. Es sei hier nicht leicht zu entscheiden zwischen einem subakuten Arzneiexanthem oder einer konfluierten Psoriasis. Temperatur nie über $38^{\circ}$ und reichliche Eosinophilie. Die Diagnose bleibt in suspenso. Nach fast völliger Heilung mittelst Eurobin zeigten sich am Rücken einigc frische Psoriasisstellen, so daß nachträglich die Diagnose: konfluierte Psoriasis gestellt werden muß.

Harmsen (aus der Arning schen Abteilung). 54jährige Frau, welche bis jetzt nie geschlecbtskrank war, 6 noch lebende und 
472 Demonstr.-Abend im Allg. Krankenh. St. Georg in Hamburg.

ein totgeborenes Kind zur Welt gebracht hat und sich vor 2 Jahren bereits in der Menopause befand. Bis 27./I. hatte sie ein Pflegekind, das dann starb, während sich bei ihr seit Ende März a. a. zuerst im Gesicht, dann über den ganzen Körper hin ein enormes, derbes pap alöses Luid entwickelte mit Condylomen ad genitalia und reichlichem Spirochaetenbefund in den Effloreszenzen; die Massigkeit der Affektion im Gesicht läßt dasselbe wie eine facies leonina erscheinen und könnte in Lepraländern den Verdacht einer Lepra erwecken.

W estberg (Hamburg). 